\title{
Mechanisms Whereby Exogenous Adenine Nucleotides Improve Rabbit Renal Proximal Function during and after Anoxia
}

\author{
L. J. Mandel, T. Takano, S. P. Soltoff, and S. Murdaugh \\ Machu Picchu Research Foundation and Department of Physiology, Duke University Medical Center, Durham, North Carolina 27710
}

\begin{abstract}
When a suspension of rabbit proximal tubules is subjected to anoxia, ATP falls by 80-90\% during 40 min of anoxia, and upon reoxygenation (reox) the cells only recover $25-50 \%$ of their initial ATP. Addition of Mg-ATP (magnesium chloridetreated ATP), Mg-ADP, or Mg-AMP (five aliquots of 200 $\mathrm{nmol} / \mathrm{ml}$ added $10 \mathrm{~min}$ apart) during anoxia causes complete recovery of ATP levels, and respiratory and transport function after $\mathbf{4 0}$ min of reox. Similar additions of adenosine (ADO), or inosine (INO), or Mg-ATP only during reox are less effective. Lactate dehydrogenase (LDH) release after $\mathbf{4 0} \mathrm{min}$ of anoxia is $30-40 \%$ under control conditions, only $10-15 \%$ when adenine nucleotides or ADO are added during anoxia, and $\mathbf{2 0 \%}$ when INO is added, suggesting that these additions may stabilize the plasma membrane during anoxia and help preserve cellular integrity. During reox, recovery may depend on the entry of ATP precursors and, therefore, we explored the mechanism whereby exogenous ATP increases the intracellular ATP content. Additions of Mg-ATP, Mg-ADP, or Mg-AMP to continuously oxygenated tubules increase cellular ATP content three- to fourfold in $1 \mathrm{~h}$. The added ATP and ADP are rapidly degraded to $A M P$, and more slowly to $A D O$, INO, and hypoxanthine. Furthermore, the ATP-induced increase in cellular ATP is abolished by the exogenous addition of adenosine deaminase, which converts extracellular ADO to INO. These results suggest that the increase in cellular ATP requires extracellular ADO. The ADO obtained from the breakdown of AMP may be preferentially transported into the renal cells to be resynthesized into cellular AMP and ATP.
\end{abstract}

\section{Introduction}

Ischemia produces a complex series of pathologic events in the kidney which, depending on their duration, leads to reversible or irreversible cell injury (1-4). One of the earliest manifestations of renal ischemia is a rapid decrease in cortical ATP content (5-7), which is depleted by $60-90 \%$ within minutes of

Address all correspondence to Dr. Lazaro Mandel, Department of Physiology, Box 3709U, Duke University Medical Center, Durham, NC 27710.

Dr. S. P. Soltoff's current address is Department of Physiology, Tufts University School of Medicine, Boston, MA 02111, and Dr. T. Takano's is Department of Hygiene, Faculty of Medicine, Tokyo Medical and Dental University, Yushima 1-5-45, Bunkyo-ku, Tokyo 113, Japan.

Received for publication 15 September 1986 and in revised form 6 November 1987.

J. Clin. Invest.

(C) The American Society for Clinical Investigation, Inc.

$0021-9738 / 88 / 04 / 1255 / 10 \quad \$ 2.00$

Volume 81, April 1988, 1255-1264 the initiation of ischemia (5-7). Initially, the decreased ATP appears as an accumulation of AMP (5-8). The AMP is further hydrolyzed in a slower reaction to mainly hypoxanthine $(\mathrm{HX})^{1}$ in the rabbit $(7,8)$ and a mixture of $\mathrm{HX}$ and xanthine in the rat $(5,6)$. Therefore, as the ischemic time is increased, there is a progressive degradation of adenine nucleotides in the cells reaching 75-90\% loss after 45-60 min (5-8). During reoxygenation, resynthesis of ATP from AMP is fast, and therefore, the cells can rapidly recover their ATP contents when the total adenylate loss is minor (5-8). Conversely, resynthesis of ATP from $H X$ is very slow $(7,9)$. Based on these considerations, it is not surprising that postischemic recovery of the kidney appears to be inversely correlated with the ischemic adenylate loss as well as the accumulation of $\mathrm{HX}$ and xanthine (10).

Various investigators have attempted to ameliorate this nucleotide loss by infusion of nucleotides or nucleosides before or after ischemia. Addition of ATP, ADP, AMP, or adenosine (ADO) at concentrations of 1-5 mM produced dramatic improvements in tissue recovery from ischemia (11-13). Inosine (INO) infusion added to a saline solution produced similar results $(14,15)$, however, the concentration used $(160 \mathrm{mM})$ was so large that the effects may be due to the hypertonicity and may not be different from the response to hypertonic mannitol $(16,17)$. All the aforementioned studies designed to ameliorate the pathological effects of ischemia were performed in vivo, where the protective mechanisms are difficult to study. In these preparations, it is particularly difficult to separate hemodynamic from tubular effects because anoxia is produced simultaneously with a hemodynamic blockage. Therefore, suspensions of proximal tubules have been used by Weinberg (18) and us (19) to enable the study of the tubular effects of anoxia independently from the other consequences of clamp-induced ischemia. However, this in vitro anoxia model differs in significant ways from in vivo-induced ischemia. For example, metabolic substrates are available in the suspension during anoxia, the $\mathrm{pH}$ is held relatively stable by buffers, and constituents lost to the medium during anoxia can be reclaimed during reoxygenation. These and other differences between the models need to be taken into account when they are compared.

In our previous studies (19) the tubules were subjected to varying times of hypoxia or anoxia, followed by reoxygenation, and the effects of these perturbations were evaluated on a number of cellular transport and respiratory parameters. We found that $20 \mathrm{~min}$ of anoxia produced reversible changes, whereas 30-40 min of anoxia caused irreversible damage, which is similar to the time frame encountered in in vivo ischemia studies (1-4). In the present communication, we report the measurements of adenine nucleotide contents and

1. Abbreviations used in this paper: ADO, adenosine; HX, hypoxanthine; IMP, inosine monophosphate; INO, inosine; LDH, lactate dehydrogenase; $\mathrm{Mg}$-ATP, magnesium chloride-treated ATP; $\mathrm{QO}_{2}$, increase in oxygen consumption. 
their breakdown products as a function of time in anoxia and oxygenated recovery. The effects of nucleotide and nucleoside addition during anoxia and under oxygenated conditions were also studied to understand the mechanisms whereby the protection from anoxia occurs.

\section{Methods}

Preparation of renal tubule suspension. Renal cortical tubules were prepared from female New Zealand White rabbits (Bunny Haven, Durham, NC; 3-4 kg wt) according to previously published methods $(20,21)$. In brief, after the animal was injected with heparin and anesthetized with ether, both kidneys were perfused at $37^{\circ} \mathrm{C}$ with a medium containing $\mathrm{NaCl}(115 \mathrm{mM}), \mathrm{NaHCO}_{3}(25 \mathrm{mM}), \mathrm{NaH}_{2} \mathrm{PO}_{4}(2 \mathrm{mM})$, $\mathrm{CaCl}_{2}(1 \mathrm{mM}), \mathrm{KCl}(5 \mathrm{mM}), \mathrm{MgSO}_{4}(1 \mathrm{mM})$, glucose $(5 \mathrm{mM})$, lactate $(4 \mathrm{mM})$, alanine ( $1 \mathrm{mM}), 0.6 \%$ dextran, and mannitol ( $25 \mathrm{mM})$ equilibrated with $95 \% \mathrm{O}_{2} / 5 \% \mathrm{CO}_{2}$ ( $\mathrm{pH} 7.4$ ). After clearing the kidneys with this solution, perfusion was continued with the same solution to which collagenase was added $(124 \mathrm{U} / \mathrm{ml})$, followed by the original solution to clear the collagenase. The cortex was then excised, minced, dispersed, and washed three times with ice-cold medium containing $\mathrm{NaCl}$ (140 $\mathrm{mM}), \mathrm{NaH}_{2} \mathrm{PO}_{4}(2 \mathrm{mM}), \mathrm{BaCl}_{2}$ (1 mM), $\mathrm{KCl}$ (5 mM), $\mathrm{MgCl}_{2}$ (1 mM), and Hepes $(10 \mathrm{mM})(\mathrm{pH} 7.4)$. The use of $\mathrm{BaCl}_{2}$, throughout the preparative steps in the cold, was instituted to obtain tubules with total calcium contents comparable to those found in the in vivo kidney (see reference 22 for details). To remove nonvital single cells and cellular debris, one of the washes involved the use of a Ficoll cushion (20). $\simeq 90 \%$ of tubules in the resulting preparation were proximal in origin and their lumens were open. The final pellet was resuspended at a concentration of 6-8 $\mathrm{mg}$ protein $/ \mathrm{ml}$ in the normal $\mathrm{NaCl}$ medium containing $\mathrm{NaCl}$ (115 mM), $\mathrm{NaHCO}_{3}(15 \mathrm{mM}), \mathrm{NaH}_{2} \mathrm{PO}_{4}(2 \mathrm{mM})$, $\mathrm{CaCl}_{2}(1 \mathrm{mM}), \mathrm{KCl}(5 \mathrm{mM}), \mathrm{MgSO}_{4}(1 \mathrm{mM})$, glucose (5 mM), lactate $(4 \mathrm{mM})$, alanine $(1 \mathrm{mM})$, glutamate $(5 \mathrm{mM})$, malate $(5 \mathrm{mM})$, valerate $(1 \mathrm{mM})$, Hepes $(10 \mathrm{mM})$, and $0.6 \%$ dextran, equilibrated with $95 \%$ $\mathrm{O}_{2} / 5 \% \mathrm{CO}_{2}$ (pH 7.4).

Anoxia experiments. Each experiment was initiated with a 20-min preequilibration of the tubule suspension at $37^{\circ} \mathrm{C}$ in the normal $\mathrm{NaCl}$ solution bubbled with $95 \% \mathrm{O}_{2} / 5 \% \mathrm{CO}_{2}$. Anoxia was achieved by closing a specially designed thermostated chamber (20), and allowing the tubules to consume all the oxygen in the solution. Anoxia was maintained for $10,20,30$, or $40 \mathrm{~min}$. After each period of anoxia, the tubule suspension was reoxygenated for 20 or $\mathbf{4 0} \mathrm{min}$ in a shaker bath under a $95 \% \mathrm{O}_{2} / 5 \% \mathrm{CO}_{2}$ atmosphere at $37^{\circ} \mathrm{C}$. Samples were taken from the suspension three times during each experiment: immediately before anoxia, immediately after anoxia, and after reoxygenation. Previous experiments (19) have shown that tubule suspensions were stable under oxygenated conditions for the duration of these experiments. Therefore, percent impairment of each function was calculated using the respective control value obtained just before anoxia. At each sampling time, nystatin-stimulated respiration, percentage of lactate dehydrogenase (LDH) released from the cells, total cellular K content, and cellular and extracellular content of adenine nucleotides, and their breakdown products were measured.

Nystatin-stimulated respiration. Previous studies in our laboratory (23) have determined that addition of nystatin to proximal tubules leads to a rapid increase in oxygen consumption $\left(\mathrm{QO}_{2}\right)$ caused by the entry of sodium from the extracellular medium and the consequent stimulation of $\mathrm{Na}, \mathrm{K}-\mathrm{ATPase}$ activity. The $\mathrm{QO}_{2}$ obtained with nystatin addition has been shown to equal the mitochondrial state three respiratory rate under normal conditions (23). Therefore, nystatin-stimulated $\mathrm{QO}_{2}$ was used to measure the maximal rate of coupled respiration obtainable in the intact tubules before and after anoxia or hypoxia. Nystatin was added at a concentration of $160 \mathrm{mg} / \mathrm{ml}$. Measurements of $\mathrm{QO}_{2}$ were made in a closed magnetically stirred thermostated chamber of $1.6 \mathrm{ml}$ in volume, using a Clark-type oxygen electrode.

Chemical assays. Total cell potassium content was measured by first layering a $0.5-\mathrm{ml}$ sample onto $0.4 \mathrm{ml}$ of a $2: 1$ mixture of dibutyl/ dioctyl phthalate in a $1.5-\mathrm{ml}$ microcentrifuge tube, which was centrifuged for at least $10 \mathrm{~s}$ using a centrifuge (5412; Eppendorf, Westbury, NY). After discarding the supernatant (in samples obtained during anoxia and reoxygenation, 5-10\% of the total protein was found in the supernatant) and the phthalate, the pellet was dissolved in $0.1 \mathrm{ml}$ of $14 \%$ perchloric acid and the potassium content was measured using an atomic absorption spectrophotometer (model 460; Perkin-Elmer Corp., Instrument Div., Norwalk, CT). Protein was measured on the same sample by the biuret method (24).

LDH was assayed according to the procedure of Bergmeyer et al. (25). Released LDH from the cells was expressed as percentage of total LDH (released LDH plus nonreleased LDH in the cells). Total LDH was measured after plasma membrane disruption by freeze thawing.

Adenine nucleotides and their breakdown products were measured using an HPLC system (Waters Associates, Millipore Corp., Milford, MA). To measure the combined nucleotide content of the cells and the extracellular medium, samples $(0.5 \mathrm{ml})$ were added to equal volumes of ice-cold, $6 \%$ perchloric acid. To measure the contents of the extracellular medium, samples $(0.5 \mathrm{ml})$ were first layered onto $0.4 \mathrm{ml}$ of a 2:1 mixture of dibutyl/dioctyl phthalate in a $1.5-\mathrm{ml}$ microcentrifuge tube, and centrifuged for at least $10 \mathrm{~s}$ using a centrifuge (5412; Eppendorf). The resulting supernatant was then added to an equal volume of $6 \%$ perchloric acid. In both of these types of samples, the supernatant remaining after the protein was precipitated by centrifugation (5412; Eppendorf), was transferred to another tube, neutralized with a small volume of $2.14 \mathrm{M} \mathrm{KOH}-1.55 \mathrm{M} \mathrm{K}_{2} \mathrm{CO}_{3}$, and centrifuged to remove the precipitate; the supernatant was stored at $-20^{\circ} \mathrm{C}$ until assayed by HPLC. A gradient system, using $100 \mathrm{mM} \mathrm{NH}_{4} \mathrm{H}_{2} \mathrm{PO}_{4}$ (pH 5.5) and methanol, with a 5- $\mu \mathrm{M} \mu$ Bondapak column (Waters Associates) was used to separate the nucleotides. This procedure permitted the quantitative measurement of ATP, ADP, AMP, ADO, INO, HX, IMP (inosine monophosphate), and xanthine (26).

Materials. Collagenase (type 4) was obtained from Sigma Chemical Co. (St. Louis, MO) or Worthington Biochemical Corp. (Freehold, NJ). Digitonin, nystatin (mycostatin), Hepes, ATP, and ADP were obtained from Calbiochem-Behring Diagnostics, American Hoechst Corp. (La Jolla, CA). Dextran T-40 and Ficoll were purchased from Pharmacia Fine Chemicals, Div. of Pharmacia (Piscataway, NJ). Ouabain, AMP, ADO, INO, and Tris were obtained from Sigma Chemical Co. All other chemicals were reagent grade. Nystatin was dissolved in dimethyl sulfoxide.

Statistics. All values are reported as the mean \pm SE. Data were analyzed by analysis of variance or Student's $t$ test. Multiple means were tested for significance using Fisher's protected, least significant difference test and a $P$ value of $<0.05$.

\section{Results}

Adenine nucleotides and their breakdown products during anoxia and reoxygenation. Fig. 1 shows that cellular levels of ATP decreased by $63 \%$ within $10 \mathrm{~min}$ of anoxia, and continued to decrease by another $27 \%$ in $40 \mathrm{~min}$. ADP levels remained fairly constant during the first 20 min of anoxia, decreasing only after $30 \mathrm{~min}$. AMP values increased rapidly as ATP was hydrolyzed, remaining fairly constant for the first 30 min of anoxia. Only the 40-min value was significantly lower. The total cellular adenine nucleotide content (ATP + ADP + AMP) declined continuously with time in anoxia to only $25 \%$ of control after $40 \mathrm{~min}$. No ADO was measurable, some INO was found mainly extracellularly (Table I), but the main breakdown product of AMP was HX, which was found mostly in the extracellular medium. As seen in Fig. 1, the HX concentration increased continuously with time in anoxia. Only after $10 \mathrm{~min}$ in anoxia did the increase in HX levels equal the decrease in total adenine nucleotides. After $20 \mathrm{~min}$ and longer 


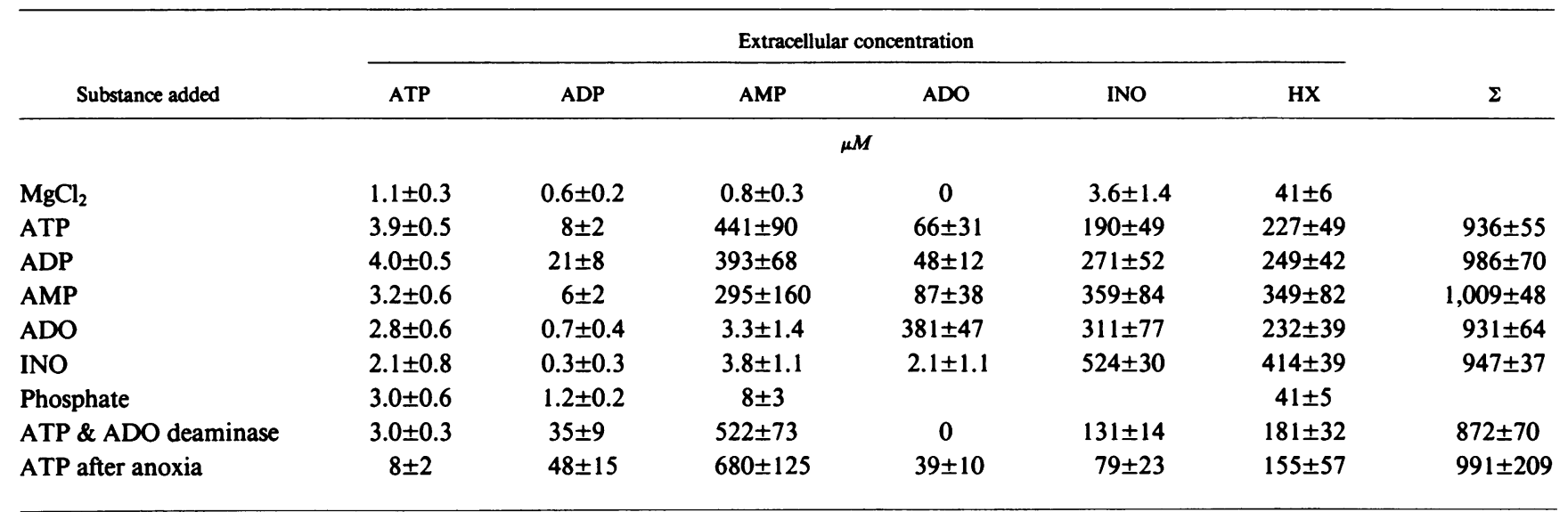

All values are means $\pm \mathrm{SEM} ; \mathrm{MgCl}_{2}$, ADP, and ATP after anoxia, $n=7 ; \mathrm{ATP}, n=5$; all other, $n=4$. Each substance was added in five aliquots, each (except for phosphate) adding a concentration of $0.2 \mathrm{mM}$ to the solution at 10 -min intervals during the 40 min of anoxia for a total addition of $1 \mathrm{mM}$. Phosphate additions were $0.6 \mathrm{mM}$ for a total of $3 \mathrm{mM}$. For the experiments shown in the last row, ATP was added in five aliquots after anoxia. All additions (except phosphate) contained an equal concentration of $\mathbf{M g C l}_{2}$.

times in anoxia, the HX levels were larger than the total decrease in adenine nucleotide content, suggesting a breakdown of other cellular constituents.

During these experiments, little, if any, ATP or ADP were found in the extracellular medium under control conditions, but some AMP was usually found there $(\sim 0.5-1.0 \mu \mathrm{M})$. After $40 \mathrm{~min}$ of anoxia, all three adenine nucleotides were detectable extracellularly at concentrations of $0.5-1.0 \mu \mathrm{M}$ (Table I). No significant amounts of ADO, xanthine, or IMP were found under any of these conditions. Furthermore, to test the identity of the HX peak in samples from anoxic tissue, xanthine oxidase was added to two random samples. This procedure caused the complete disappearance of the HX peak and the appearance of a new xanthine peak in the HPLC traces from both samples.

Recovery from anoxia was examined in paired experiments in which tubules were exposed to 20 or $\mathbf{4 0}$ min of anoxia. As shown in Fig. $2 \mathrm{~A}$, the ATP levels increased from $27 \%$ of control immediately after $20 \mathrm{~min}$ of anoxia to $76 \%$ of con-

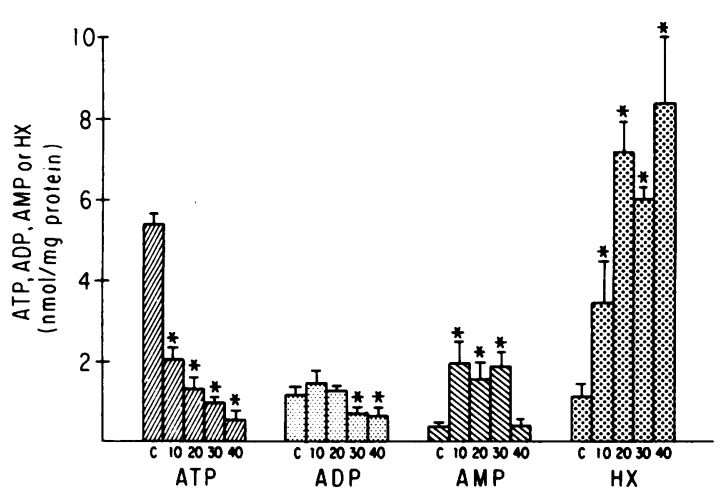

Figure 1. Cellular content of ATP, ADP, AMP, and HX as a function of time in anoxia. $C=$ control; $10,20,30$, and 40 are the times in minutes that the tubules were subjected to anoxia, respectively $(n$ $=4)$. ${ }^{*}$ Significantly different from control. trol after 20 min of oxygenated recovery. The large decrease in AMP levels (to $\sim 0.0$ ) accounted stoichiometrically for most of the ATP recovery, the rest was probably due to resynthesis from other sources that included HX. Complete ATP recovery was achieved after $40 \mathrm{~min}$ of reoxygenation, and this was accompanied by a small but significant decline in HX. Note that the AMP level remained depressed even after $40 \mathrm{~min}$ of recovery.

The pattern of recovery was very different after $40 \mathrm{~min}$ of anoxia, as shown in Fig. $2 \mathrm{~B}$. Reoxygenation for $20 \mathrm{~min}$ produced recovery of ATP from $10 \%$ to $27 \%$ of control and an additional $20 \mathrm{~min}$ of reoxygenation only increased this value to $34 \%$ of control. The ADP and AMP levels also remained depressed, the latter becoming almost unmeasurable during recovery. Again, much of the ATP recovery could be attributed to the further decrease in AMP levels during this period.
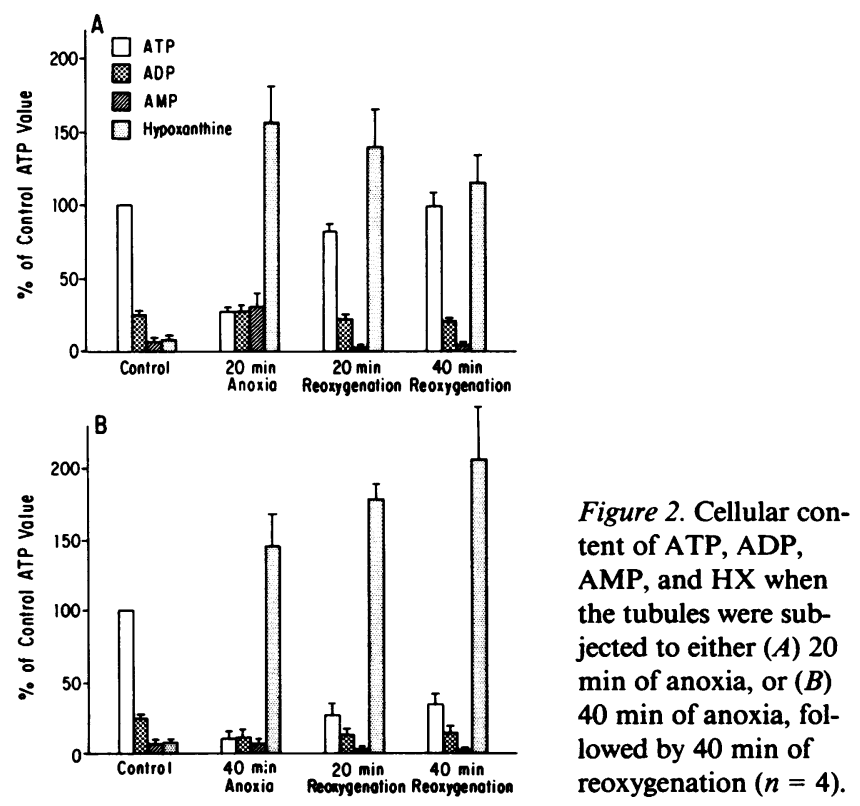
The HX level was already $45 \%$ larger than the control ATP value after $40 \mathrm{~min}$ of anoxia. During recovery, the HX level continued to increase, in contrast to the behavior observed during the recovery from $20 \mathrm{~min}$ of anoxia (Fig. $2 \mathrm{~A}$ ). The continued production of HX suggests that the cells may be undergoing a generalized breakdown, leading to irreversible cell injury (see Discussion).

Addition of magnesium chloride-treated ATP (Mg-ATP) or its hydrolysis products during anoxia. A series of paired experiments was performed to determine the effects of Mg-ATP addition on adenine nucleotides and their breakdown products during $\mathbf{4 0} \mathrm{min}$ of anoxia followed by $\mathbf{4 0} \mathrm{min}$ of reoxygenation. Mg-ATP was added in five aliquots, each one adding a concentration of $0.2 \mathrm{mM}$ to the solution at 10 -min intervals during the $40 \mathrm{~min}$ of anoxia. As seen in Fig. 3, addition of extracellular Mg-ATP did not change the measured value of intracellular ATP after $40 \mathrm{~min}$ of anoxia. However, there were significant increases in the ATP recoveries obtained after 20 min and $40 \mathrm{~min}$ of reoxygenation. The addition of Mg-ATP during anoxia produced an $82 \pm 11 \%$ recovery of ATP level after $20 \mathrm{~min}$ and $157 \pm 12 \%$ recovery after $40 \mathrm{~min}$ of reoxygenation.

An analysis of the extracellular medium composition was made during these experiments in an attempt to determine whether ATP or its breakdown products were responsible for the improved recovery. As shown in Fig. 4, little ATP was found extracellularly at any time point. Only $20 \mu \mathrm{M}$ of extracellular ATP was found after $20 \mathrm{~min}$ of anoxia, despite the total addition of $400 \mu \mathrm{M}$ up to that time point, demonstrating that a fast hydrolysis of ATP occurred extracellularly. Although the extracellular ADP values increased significantly, most of the added ATP was found hydrolyzed to AMP, ADO, INO, and HX. ADO and INO displayed similar time courses to those of $\mathrm{HX}$ and therefore were not shown in Fig. 4 for clarity. The extracellular concentrations of ATP and its main breakdown products after $40 \mathrm{~min}$ of anoxia and reoxygenation are shown in Table I. IMP could also be detected in some experiments, but its concentration remained below $20 \mu \mathrm{M}$.

The rapid hydrolysis of extracellular ATP made it difficult to identify the compound(s) responsible for the improved recovery from anoxia. Therefore, a series of experiments was performed to test the effects that extracellular addition of each hydrolytic product had on the recovery from anoxia. Each of the compounds was added in five aliquots during anoxia using an experimental protocol identical to that used for the $\mathrm{Mg}$ ATP additions. Each substance was added in a 1:1 combina-

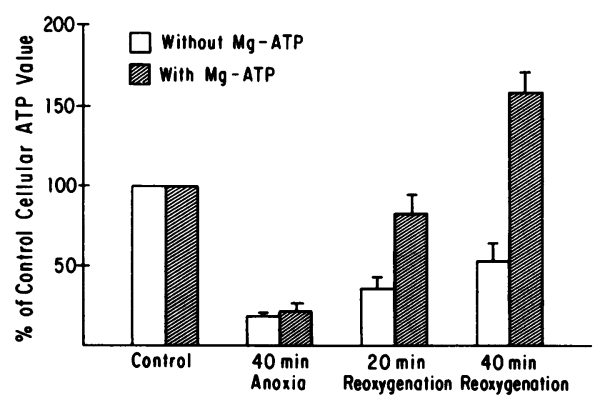

Figure 3. Cellular ATP contents of tubules exposed to $40 \mathrm{~min}$ of anoxia followed by $40 \mathrm{~min}$ of reoxygenation. Paired studies $(n=5)$ comparing the effects of external Mg-ATP addition during anoxia.

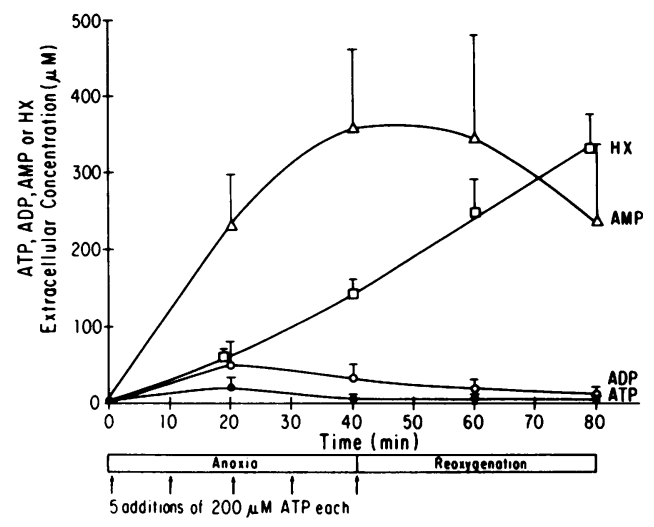

Figure 4. Extracellular concentrations of ATP, ADP, AMP, and HX during anoxia and reoxygenation when the tubule suspension was subjected to five exogenous additions of ATP at the times indicated by the arrows $(n=4)$.

tion with $\mathrm{Mg}-\mathrm{Cl}_{2}$ and the control conditions received five additions of $0.2 \mathrm{mM} \mathrm{MgCl}$. The one exception was $\mathrm{NaH}_{2} \mathrm{PO}_{4}$ (titrated to $\mathrm{pH}$ 7.4), which was added in five aliquots that increased the solution concentration by $0.6 \mathrm{mM}$ each. The rate of breakdown of each of the added compounds was similar to that shown in Fig. 4. The extracellular composition after 40 min of anoxia and $40 \mathrm{~min}$ of reoxygenation for each of the additions is shown in Table I. In these experiments the sum of all the measured breakdown products always accounted for 93-100\% of the added substance, as shown in Table I. Added ADP was rapidly hydrolyzed to AMP and further breakdown products. Added AMP was metabolized less rapidly than ATP or ADP, as would be expected from Fig. 4. Its main breakdown products were INO and HX. Added ADO was converted to INO and HX, and much of the added INO was found in HX.

As seen in Table II, additions of ATP, ADP, or AMP during anoxia offered the most substantive protection of cellular ATP content, producing excellent recovery after $20 \mathrm{~min}$ and supramaximal recovery after $\mathbf{4 0} \mathrm{min}$ of reoxygenation. ADO produced significant improvement in the recovery of cellular ATP content, but after $\mathbf{4 0}$ min of reoxygenation, this content was significantly lower than that achieved by nucleotide additions. INO addition did not provide any protection as compared with controls.

Comparison of the last two rows of Table II offers some insight into the separate roles of extracellular adenine nucleotide aliquots added during anoxia vs. those added after anoxia, during reoxygenation. At 20 min of reoxygenation, the ATP added after anoxia produced no significant improvement in cellular ATP content, whereas the samples to which ATP was added during anoxia, which was followed by a wash, had a significantly higher cellular ATP content. The latter ATP content was not different from that achieved by the cells with nucleotide additions during anoxia and were not washed before reoxygenation. These results highlight the protection offered by extracellular ATP addition during anoxia and the relatively small role played by the extracellular nucleotides during the first 20 min of reoxygenation. In contrast, the presence of extracellular adenine nucleotides produced a signifcant increase in cellular ATP content between 20 and $40 \mathrm{~min}$ of reoxygenation, although the increase was less than in the ATP, ADP, or AMP groups. 
Table II. Effect of Added Adenine Nucleotides and Their Breakdown Products on Cellular ATP

Content and LDH Release during Anoxia and Reoxygenation

\begin{tabular}{|c|c|c|c|c|c|c|}
\hline Substance added & \multicolumn{3}{|c|}{ ATP content } & \multicolumn{3}{|c|}{ LDH released } \\
\hline & \multicolumn{3}{|c|}{ \% control } & \multicolumn{3}{|c|}{$\%$} \\
\hline $\mathrm{MgCl}_{2}$ & $17 \pm 2$ & $33 \pm 5$ & $46 \pm 7$ & $5 \pm 1$ & $35 \pm 3$ & $40 \pm 4$ \\
\hline ATP & $23 \pm 4^{*}$ & $82 \pm 11^{*}$ & $164 \pm 12^{* \ddagger}$ & $6 \pm 1$ & $16 \pm 4^{*}$ & $19 \pm 5^{*}$ \\
\hline ADP & $28 \pm 2^{*}$ & $103 \pm 21^{* \pm}$ & $191 \pm 27^{* \ddagger}$ & $5 \pm 1$ & $19 \pm 3^{*}$ & $24 \pm 4^{*}$ \\
\hline ADO & $18 \pm 2$ & $61 \pm 8^{*}$ & $93 \pm 13^{*}$ & $5 \pm 1$ & $14 \pm 1^{*}$ & $19 \pm 2^{*}$ \\
\hline INO & $17 \pm 3$ & $35 \pm 3$ & $53 \pm 7$ & $5 \pm 2$ & $20 \pm 1^{*}$ & $35 \pm 4$ \\
\hline ATP after anoxia & $23 \pm 4^{*}$ & $46 \pm 10$ & $77 \pm 14^{*}$ & $6 \pm 2$ & $35 \pm 5$ & $43 \pm 4$ \\
\hline ATP + wash & $35 \pm 6^{*}$ & $81 \pm 13^{*}$ & $103 \pm 10^{*}$ & $7 \pm 1$ & $20 \pm 3^{*}$ & $4 \pm 1^{\text {*\# }}$ \\
\hline
\end{tabular}

All values are means \pm SEM. ${ }^{* \ddagger}$ Significantly different from each other $(P<0.05)$. Numbers of experiments and additions were as described in Table I. Control values for ATP were obtained immediately before anoxia for each experiment (see Methods). In the "ATP + wash" experiments, ATP was added in five aliquots during anoxia followed by rapid washing of the cells and resuspension in fresh oxygenated medium containing no nucleotides or nucleosides; anoxia samples were obtained before the washing procedure; $n=4$ for these experiments. Reox., reoxygenation.

The improvements in ATP recovery were also mirrored in the preservation of cellular LDH content, which is also seen in Table II. Addition of any of the nucleotides or nucleosides significantly decreased the percentage of LDH released to the extracellular medium during anoxia. No additional significant release of LDH could be detected during reoxygenation, as shown previously by Takano et al. (19), although a small release may be obscured by the LDH lost from the cells during anoxia. The one group of experiments in which the cells were washed after anoxia ("ATP + wash") showed a release of $4 \pm 1 \%$ LDH during reoxygenation, which may be representative of the amount released under other conditions, as well. The only exception was INO, which protected during anoxia, but not upon reoxygenation. Not surprisingly, ATP addition after anoxia was not different from control. The present results measuring LDH release at these time points are particularly important because they demonstrate two forms of protective action by adenine nucleotides and nucleosides: $(a)$ protection of plasma membrane integrity (less LDH release) during anoxia, and $(b)$ serving as a source of intracellular adenine nucleotide precursors during reoxygenation (see Discussion).

Addition of ATP, ADP, or AMP produced almost complete recovery of nystatin-stimulated respiration and $\mathrm{K}$ contents of the tubules after $40 \mathrm{~min}$ of reoxygenation, as shown in Table III. The presence of the nucleotides during anoxia was clearly the important time for the protective action, since washing the cells before reoxygenation did not alter the course of recovery (Table III, last row). ADO improved only the nystatin-stimulated respiration but did not significantly elicit better recovery in $\mathrm{K}$ contents than $\mathrm{MgCl}_{2}$ addition did. INO and ATP added after anoxia did not significantly improve recovery of either of these variables.

To separate further the effects of ATP itself from those of its hydrolytic products, a series of five experiments was performed, with results shown in Table IV. Addition of phosphate (another hydrolytic product of ATP) produced no protection from anoxia, as compared with the $\mathrm{MgCl}_{2}$ controls. Paired experiments were performed to test the effects of ADO deami- nase on ATP addition during anoxia. As seen in Table $\mathrm{I}$, the addition of $5 \mu \mathrm{g} / \mathrm{ml}$ ADO deaminase converted all extracellular ADO to INO without affecting the concentrations of the other ATP breakdown products. Addition of ATP during anoxia provided protection and enhanced recovery in all the measured variables in the presence and absence of ADO deaminase. These experiments demonstrate that ADO is not required for preservation of plasma membrane integrity during anoxia. However, a difference was seen in the ATP recovery from anoxia when each value in the absence of deaminase was

Table III. Effect of Added Nucleotides and Their Breakdown Products on Nystatin-stimulated Respiration and Cellular $K$ Content during Anoxia and Reoxygenation

\begin{tabular}{llllc}
\hline & $\begin{array}{c}\text { Nystatin- } \\
\text { stimulated } \\
\mathrm{QO}_{2}\end{array}$ & & \multicolumn{2}{c}{ K content } \\
\cline { 2 - 3 } \cline { 4 - 5 } Substance added & 40 min reox. & & 40 min anoxia & 40 min reox. \\
\hline & $\%$ control & & \multicolumn{2}{c}{$\%$ control } \\
$\mathrm{MgCl}_{2}$ & $53 \pm 7$ & & $36 \pm 3$ & $80 \pm 6$ \\
$\mathrm{ATP}$ & $97 \pm 13^{*}$ & & $33 \pm 2$ & $112 \pm 7^{*}$ \\
ADP & $74 \pm 7^{*}$ & & $32 \pm 2$ & $94 \pm 5^{*}$ \\
AMP & $88 \pm 9^{*}$ & & $31 \pm 2$ & $103 \pm 7^{*}$ \\
ADO & $82 \pm 10^{*}$ & & $32 \pm 2$ & $88 \pm 14$ \\
INO & $63 \pm 7$ & & $30 \pm 2$ & $80 \pm 10$ \\
ATP after anoxia & $55 \pm 5$ & & $33 \pm 5$ & $79 \pm 5$ \\
ATP + wash & $83 \pm 10^{*}$ & & $32 \pm 2$ & $100 \pm 4^{*}$ \\
& & &
\end{tabular}

All values are means \pm SEM.

* Significantly different from each other $(P<0.05)$. Numbers of experiments and additions were as described in Table I. Control values for each variable were obtained immediately before anoxia for each experiment (see Methods). Control values for nystatin-stimulated $\mathrm{QO}_{2}$ averaged $41.7 \pm 2.5 \mathrm{nmol} / \mathrm{min} . \mathrm{mg}$ protein; for $\mathrm{K}$ content, control values averaged $250 \pm 4 \mathrm{nmol} / \mathrm{mg}$ protein. Reox., reoxygenation. 
Table IV. Effect of Added Phosphate, ATP, or ATP Plus ADO Deaminase on Cellular ATP Content, LDH Release, Nystatin-stimulated Respiration, and $K$ Content during Anoxia and Reoxygenation*

\begin{tabular}{|c|c|c|c|c|c|c|c|c|}
\hline \multirow[b]{2}{*}{ Substance added } & \multicolumn{2}{|c|}{ ATP content } & \multicolumn{3}{|c|}{ LDH released } & \multirow{2}{*}{$\begin{array}{c}\begin{array}{c}\text { Nystatin-stimulated } \\
\mathrm{QO}_{2}\end{array} \\
40 \mathrm{~min} \text { reox. }\end{array}$} & \multicolumn{2}{|c|}{$\mathrm{K}$ content } \\
\hline & $\begin{array}{l}40 \mathrm{~min} \\
\text { anoxia }\end{array}$ & $\begin{array}{l}40 \text { min } \\
\text { reox. }\end{array}$ & Control & $\begin{array}{l}40 \text { min } \\
\text { anoxia }\end{array}$ & $\begin{array}{l}40 \text { min } \\
\text { anoxia }\end{array}$ & & $\begin{array}{l}40 \mathrm{~min} \\
\text { anoxia }\end{array}$ & $\begin{array}{l}40 \mathrm{~min} \\
\text { reox. }\end{array}$ \\
\hline & \multicolumn{2}{|c|}{ \% control } & \multicolumn{3}{|c|}{$\%$} & $\%$ control & \multicolumn{2}{|c|}{ \% control } \\
\hline $\mathrm{MgCl}_{2}$ & $14 \pm 2$ & $33 \pm 5$ & $6 \pm 1$ & $47 \pm 4$ & $52 \pm 5$ & $37 \pm 5$ & $38 \pm 4$ & $64 \pm 5$ \\
\hline Phosphate & $18 \pm 3$ & $28 \pm 6$ & $5 \pm 1$ & $39 \pm 5$ & $50 \pm 6$ & $35 \pm 5$ & $37 \pm 2$ & $62 \pm 4$ \\
\hline ATP & $27 \pm 5^{*}$ & $125 \pm 29^{*}$ & $6 \pm 1$ & $21 \pm 3^{*}$ & $24 \pm 3^{*}$ & $82 \pm 6^{*}$ & $36 \pm 2$ & $98 \pm 5^{*}$ \\
\hline ATP + ADO deaminase & $31 \pm 11$ & $79 \pm 15^{*}$ & $6 \pm 1$ & $22 \pm 5^{*}$ & $26 \pm 4^{*}$ & $77 \pm 9^{*}$ & $29 \pm 3$ & $86 \pm 5^{*}$ \\
\hline
\end{tabular}

All values are means $\pm \operatorname{SEM}(n=5)$. * Significantly different from their respective $\mathrm{MgCl}_{2}$ control $(P \leq 0.05)$. Additions were as described in Table I. Reox., reoxygenation.

divided by its paired value in the presence of the enzyme. In four paired experiments this ratio of ATP values was $2.2 \pm 0.5$ $(P<0.05)$, demonstrating less ATP recovery in the absence of extracellular ADO.

Addition of Mg-ATP or its hydrolysis products during normoxia. Mg-ATP or its hydrolysis products were added to the tubule suspension under normoxic conditions to understand better the nature of their protective effects during anoxia. The additions were made in five aliquots at 10-min intervals in an identical fashion to those made during anoxia. Fig. 5 shows the rapid breakdown of added extracellular ATP and the consequent accumulation of extracellular AMP. The appearance of HX under these conditions is notably slower than that seen during anoxia (Fig. 4). Similarly added ADP is rapidly hydrolyzed to AMP (results not shown) and added AMP is degraded more slowly than during anoxia, as expected from the results seen in Fig. 5.

The effects of each of these additions on the cellular ATP contents is shown in Fig. 6. Additions of ATP, ADP, or AMP increased cellular ATP levels to $20-30 \mathrm{nmol} / \mathrm{mg}$ protein in 60 min representing a three- to fourfold increase from control values. ADO caused a doubling in ATP values within the same time period, whereas INO and HX caused no significant change as compared with timed controls with no additions (not shown). The rapid hydrolysis of added ATP or ADP into

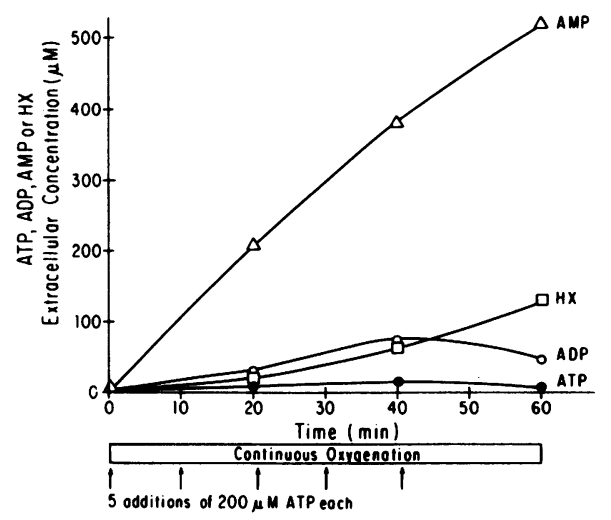

Figure 5. Extracellular concentrations of ATP, ADP, AMP, and HX during continuous oxygenation in the presence of five exogenous additions of ATP at the times indicated by the arrows $(n=4)$.
AMP and the similarities in the effects of all three adenine nucleotides suggested that they may all be due to the accumulated extracellular concentration of AMP. To ascertain the feasibility of this possibility, the intracellular ATP contents were plotted as a function of the extracellular AMP concentration, as shown in Fig. 7. The individual experimental points were obtained at the $20-$, $40-$, or 60 -min time points from different experiments in which either ATP, ADP, or AMP was added to the extracellular medium. The good correlation between these two variables suggests that AMP itself, or a breakdown product of AMP, may be preferentially transported across the plasma membrane into the cells to be synthesized into ATP.

To discriminate between these two possibilities, a series of experiments was performed in which ADO deaminase (5 $\mu \mathrm{g} / \mathrm{ml}$ ) was present in the medium before the addition of $\mathrm{Mg}$ ATP. The extracellular medium was analyzed in each of these experiments and was found to contain no measurable ADO. Results from paired experiments (Fig. 8) show a significant

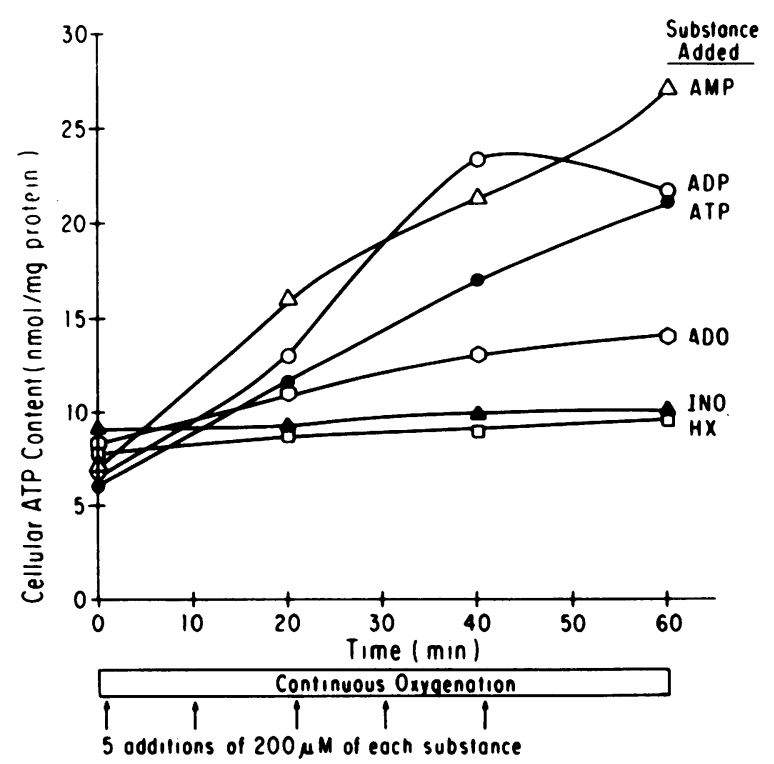

Figure 6. Cellular ATP contents during continuous oxygenation upon addition of the indicated compounds at the times shown by the arrows $(n=4)$. 


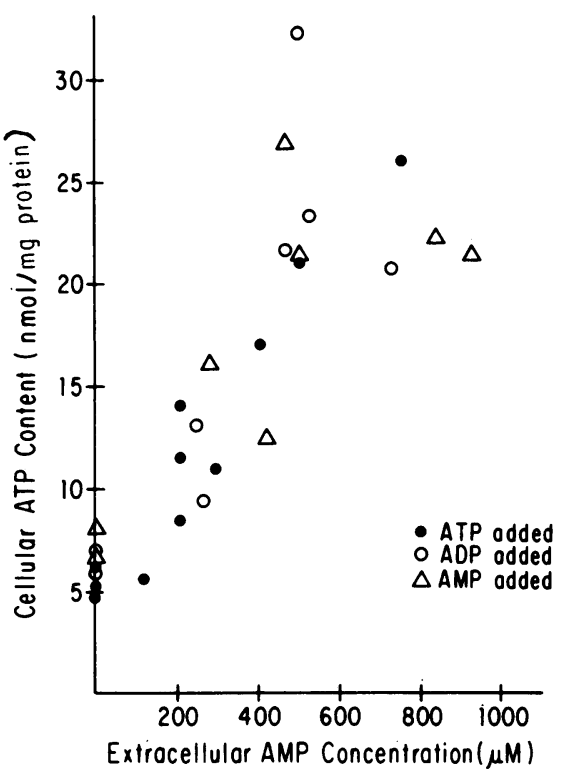

Figure 7. Cellular ATP content plotted as a function of extracellular AMP concentration when either ATP, ADP, or AMP was added to the medium during continuous oxygenation.

inhibition of the cellular ATP rise that occurred upon the addition of ADO deaminase. In this case, cellular ATP increased slowly with time, at the same rate as that of timed controls with no additions (results not shown), or as that of cells to which INO or HX had been added (Fig. 6). ADO deaminase addition did not alter the extracellular concentrations of AMP ( $434 \pm 140 \mu \mathrm{M}$, control vs. $458 \pm 170 \mu \mathrm{M}$, deaminase), but almost doubled the INO concentration $(72 \pm 22 \mu \mathrm{M}$, control, vs. $120 \pm 17 \mu \mathrm{M}$, deaminase). These results suggest that AMP itself may not be transported across the cell membrane but ADO obtained from AMP breakdown may be preferen-

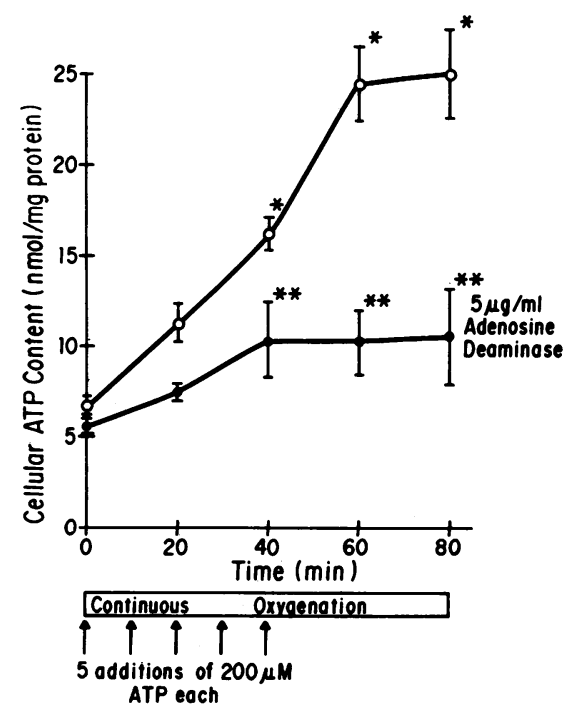

Figure 8. Cellular ATP content as a function of time in the presence and absence of ADO deaminase. ATP additions are indicated by the arrows $(n=4)$. Single asterisk indicates significant difference from value at time zero. Double asterisk indicates significant difference from paired time control without ADO deaminase. tially transported into the cell to be resynthesized into AMP and ATP (see Discussion).

\section{Discussion}

Numerous investigators have shown that clamp-induced ischemia rapidly reduces the ATP cellular content of the renal cortex (5-7). Similar results were obtained by Weinberg (18) when he subjected a suspension of proximal tubules to oxygen deprivation. The present results confirm this finding and, in addition, measure the time course for the decline in ATP and the fate of its major hydrolysis products in proximal tubules. In this tubule suspension, ATP falls $63 \%$ within the first 10 min of anoxia, and continued its decline more slowly until 90\% of ATP was lost in $40 \mathrm{~min}$ (Fig. 1). Given the rapid turnover of ATP in these cells ( $7 \mathrm{~s}$ [27]) and their low capacity for anaerobic glycolysis (28), it is perhaps surprising that the ATP levels did not decrease faster upon reaching anoxia. Two possible explanations for the slower decline might be $(a)$ mitochondrial ATP may fall more slowly than cytosolic ATP during anoxia, as recently suggested by Aw and Jones' (29) work dealing with hepatocytes. Since $\sim 30 \%$ of cellular ATP seems to be located within the mitochondria in proximal tubules (30), this could account for the slower decline in $~ 30 \%$ of the ATP between 10 and $40 \mathrm{~min}$ of anoxia and/or $(b)$ active transport work declines with ATP content (31), slowing down the rate of ATP utilization. With reduced work, anaerobic glycolysis might be able to maintain the observed ATP values.

During anoxia, ATP was rapidly hydrolyzed to AMP which, in turn, was hydrolyzed further appearing mainly as HX. This would be expected from the known properties of 5 -nucleotidase which is the main enzyme responsible for AMP hydrolysis (32). This enzyme is activated by low levels of ATP and high AMP levels (32). The activation of this enzyme is probably responsible for the continuous degradation of adenine nucleotides during anoxia. Extended times in anoxia caused large losses in total adenine nucleotide content, reaching a 75\% decline after $40 \mathrm{~min}$. No ADO was detectable under these conditions and only small concentrations of INO and IMP were detected, clearly identifying $\mathrm{HX}$ as the main degradation product. No xanthine was detectable even after reoxygenation, which is consistent with the low concentration of xanthine oxidase present in the rabbit kidney $(33,34)$.

The contrast between the reversibility obtained after 20 min of anoxia and the irreversibility after $\mathbf{4 0} \mathrm{min}$ was striking. The differences between these two responses provide important information on some early events that may lead to irreversible cell damage. The large loss of total adenine nucleotides observed after $40 \mathrm{~min}$ of anoxia may be one of the most important factors leading to irreversibility, since the low adenine nucleotide content prevents much recovery in ATP after reoxygenation. This possibility has been raised previously by Buhl (10), who found an inverse linear correlation between the accumulation of HX during ischemia in the rabbit kidney in vivo and the reversibility of ischemia. In our preparation, this nucleotide loss is also temporally correlated with the degradation of plasma membrane integrity, as measured by LDH release. The temporal pattern of HX content during anoxia and recovery is particularly informative. After $20 \mathrm{~min}$ of anoxia, HX levels decline during the reoxygenation period, probably by resynthesis into ATP. After $\mathbf{4 0} \mathrm{min}$ of anoxia, HX levels 
continue to increase during reoxygenation, suggesting a continuation of the degradative processes initiated during anoxia. It is presently unclear what the source of the HX is, however, it could originate from a number of possible cellular constituents, in particular, RNA (8). In this manner, the HX levels during reoxygenation may provide an early indication of either reversibility, when the degradative processes are halted and HX levels decline, or irreversibility, when the degradative processes continue.

Various investigators have demonstrated that infusion of adenine nucleotides protect the kidney from the pathological effects of ischemia $(11,12)$. However, the mechanism(s) whereby this protection occurs has been difficult to ascertain in the intact kidney. The preparation used in the present studies has allowed the investigation of some of the tubular mechanisms involved in this protective action. In this study all nucleotide and nucleoside additions were made in a 1:1 combination with $\mathrm{MgCl}_{2}$ to duplicate the condition used for the in vivo ischemia experiments $(11,12)$. A recent study by Weinberg (18) shows that the $\mathrm{MgCl}_{2}$ itself is not needed for some of the effects of added adenine nucleotides. However, this was not explored in the present studies. Exogenous ATP and ADP were rapidly degraded to AMP and further hydrolytic products, as also appears to occur in a variety of cells $(35,36)$. It is noteworthy that exogenous AMP hydrolysis occurred much faster during anoxia than under oxygenated conditions (compare Fig. 4 with Fig. 5), which is consistent with the properties of 5'-nucleotidase (32). Thus, it is possible that this enzyme is involved in the hydrolysis of extracellular AMP as well as intracellular AMP, as discussed earlier. This could occur either through the transport of AMP into the cells, and/or through the presence of this or a similar enzyme on the extracellular surface of the plasma membrane. Most of the AMP when broken down accumulates as INO and HX, with less appearing as ADO. As shown in Table I, the six measured compounds accounted for virtually $100 \%$ of the added substance.

As shown in a previous publication (19), much of the cellular damage occurs in the tubules during anoxia. Most of the LDH release from the cells occurs during anoxia, whereas reoxygenation leads to an apparent resealing of the plasma membrane (19). Similarly, mitochondrial function is profoundly impaired immediately after anoxia, but recovers partially after reoxygenation $(18,19)$. Therefore, the protective effects of the added nucleotides and nucleosides need to be examined in terms of their separate effects during anoxia and during reoxygenation.

We have previously shown that ATP addition protects the plasma membrane during anoxia, as evidenced by a dramatic decrease in LDH release (19). The rapid breakdown in extracellular ATP suggests that ATP itself may not be the protective agent, and similar considerations apply to ADP. The identical protective action of added ATP, ADP, and AMP, as well as the rapid breakdown of ATP and ADP into AMP, suggest that all three of these compounds elicit their protective effects through their common breakdown product, namely, AMP. However, ADO also causes $\mathrm{LDH}$ release to decrease to the same extent as the adenine nucleotides, and therefore, it is also possible that ADO itself may be the protective agent, although the experiments with ATP and ADO deaminase clearly demonstrate that the presence of ADO is not required to protect the plasma membrane during anoxia. These compounds could elicit a direct protective action from the extracellular medium, but the nature of the protective mechanism is presently unknown. On the other hand, it is likely that the extracellular presence of these added compounds will cause intracellular changes that elicit the protective action. Since intracellular ATP levels after 40 min of anoxia are only minimally affected by any of the added substances (Table II), the protection does not seem to occur at this step. Intracellular ADP levels do not appear to change either (results not shown). Unfortunately, intracellular AMP values could not be obtained in the presence of high extracellular AMP concentrations, but these are likely to be elevated, which has been shown by other investigators (6). Thus, a possible mechanism through which added adenine nucleotides might be protective is through preservation of cellular AMP levels during anoxia. However, intracellular AMP levels after $40 \mathrm{~min}$ of anoxia were unaltered by ADO or INO addition (results not shown), suggesting that the protective action of ADO did not occur through AMP. This is clearly an area that will require further investigation.

During reoxygenation, the presence of adenine nucleotides in the extracellular medium (mainly AMP) promotes almost full recovery of oxygen consumption and $\mathrm{K}$ contents (Table III) and supramaximal recovery of ATP content (Table II). This supramaximal recovery appeared to depend on the presence of ADO in the extracellular medium, since addition of ADO deaminase to eliminate ADO inhibited ATP recovery (Table IV). The addition of ATP was required only during anoxia, since a wash before reoxygenation did not alter the recovery course. Conversely, addition of ATP after anoxia brought about a much poorer recovery in all of these variables, indicating that the protection afforded by the adenine nucleotides during anoxia was the most important factor in the recovery process. An interesting contrast is provided by ADO, which protected the plasma membrane integrity during anoxia, but provided less overall recovery during reoxygenation as compared with the adenine nucleotides. A similar result was recently obtained by Sumpio et al. (37) for the intact kidney. The slower recovery may possibly be due to the slower resynthesis of ATP obtained from ADO, as compared with AMP (see below). INO was without effect, suggesting that the protection was specific for adenine nucleotides and nucleosides. Unfortunately, in these experiments it was not possible to evaluate whether the overall breakdown of cellular constituents into $\mathrm{HX}$ was halted, since all the added substances themselves were hydrolyzed to $\mathrm{HX}$.

The experiments in which the additions were made during normoxia provided additional information concerning the recovery process from anoxia. Again, the similarity of the effects of ATP, ADP, or AMP, and the rapid breakdown of the former two into AMP, suggests that their common effects may be mediated through AMP. Further support for this idea comes from Fig. 7, which shows a rough correlation between intracellular ATP values and extracellular AMP concentration. However, the results of the ADO deaminase experiments suggest that AMP itself may not be transported across the plasma membrane. The same conclusion was previously reached by Weinberg et al. (38) who used a variety of nucleotide and nucleoside metabolism and transport inhibitors on proximal renal tubules. Similar results have also been obtained by Parker (35) in red blood cells, Sasaki et al. (39) in the liver, and Frick and Lowenstein (40) in the heart. These investigators also found that the addition of adenine nucleotides increased cellular ATP levels faster than ADO addition. The explanation 
for this result is presently unclear, but several possibilities exist, namely: (a) ADO itself may inhibit ADO kinase, thereby slowing down the resynthesis of ADO into AMP at high ADO concentrations $(38,41)$. (b) The $K_{\mathrm{m}}$ of ADO for the kinase is much lower than its $K_{\mathrm{m}}$ for the deaminase. In that way, at low ADO concentrations, most of the ADO would react with the kinase, whereas at higher concentrations it would react with the deaminase (41). (c) The ADO produced by breakdown from AMP, possibly through an ecto-5'-nucleotidase (42), may be preferentially transported into the cells $(39,40)$. The transport of ADO into proximal renal tubules has been clearly demonstrated (43). Similarly, INO and HX have been shown to be transported into renal cells (44). However, the lack of significant increase in ATP levels after their addition, suggests that synthesis of ATP from these precursors is normally slow.

In conclusion, exogenous addition of adenine nucleotides during anoxia protects the function of proximal tubules in at least two ways: (a) during anoxia, it protects the plasma membrane from disruption. The mechanism for this effect is unclear. (b) During reoxygenation, it provides precursors for rapid ATP formation by transport of ADO into the cells followed by resynthesis into cellular AMP and ATP.

\section{Acknowledgments}

Dr. Takano was supported during his stay at Duke University by a grant from the Japanese Ministry of Education. Dr. Mandel would like to thank Dr. John Parker for suggesting the ADO deaminase experiments and Dr. Joel Weinberg for sharing his thoughts and unpublished observations on the lack of AMP transport into these cells. This work was supported by National Institutes of Health grant AM-26816 to Dr. Mandel. We would like to thank Dr. William Jacobs and Ms. Maria Sgambati for their help with some of these experiments, Mr. Jeff Leiser for help with the statistics, and Ellen Eatmon, Gay Blackwell, and Jinny Shelton for typing the manuscript.

\section{References}

1. Venkatachalam, M. A., D. B. Bernard, J. F. Donohoe, and N. G. Levinsky. 1978. Ischemic damage and repair in the rat proximal tubule: differences among the $S_{1}, S_{2}$ and $S_{3}$ segments. Kidney Int. 14:31-49.

2. Trump, B. F., I. K. Berezesky, and R. A. Cowley. 1982. The cellular and subcellular characteristics of acute and chronic injury with emphasis on the role of calcium. In Pathophysiology of Shock, Anoxia, and Ischemia. R. A. Cowley and B. F. Trump, editors. Williams \& Wilkins, Baltimore. 6-46.

3. Johnston, P. A., H. Rennke, and N. G. Levinsky. 1984. Recovery of proximal tubular function from ischemic injury. Am. J. Physiol. 246:F159-F166.

4. Wilson, D. R., P. E. Arnold, T. J. Burke, and R. W. Schrier. 1984. Mitochondrial calcium accumulation and respiration in ischemic acute renal failure in the rat. Kidney Int. 25:519-526.

5. Thorn, W., and F. Liemann. 1961. Metabolitkonzentrationen in de Niere und Paraaminohippursaureclearance nach akuter Ischamie und in der Erholung nach Ischamie. Pfluegers Arch. Eur. J. Physiol. 273:528-542.

6. Gerlach, E., B. Deuticke, and R. H. Dreisbach. 1963. Zum Verhalten von Nucleotiden und ihren Dephosphorylierten Abbauprodukten in der Niere bei Ischamie und kurzzeitiger Postischamischer Wiederdurchblutung. Pfluegers Arch. Eur. J. Physiol. 278:296-315.

7. Buhl, R. 1982. Purine metabolism in ischaemic kidney tissue. Dan. Med. Bull. 29:1-26.

8. Busch, E. W., I. M. Von Borcke, and B. Martinez. 1968. Abbau- wege und Abbaumuster der Purinnucleotide in Herz-, Leber-, und Nierengewebe von Kaninchen nach Kreislauf-stillstand. Biochim. Biophys. Acta. 166:546-556.

9. Gerlach, E., P. Marko, H.-G. Zimmer, I. Pechan, and Ch. Trendelenburg. 1970. Different response to adenine nucleotide synthesis de novo in kidney and brain during aerobic recovery from anoxia and ischemia. Experientia (Basel). 27:876-878.

10. Buhl, M. R. 1979. The predictive value of 5'-adenine nucleotide depletion and replenishment in ischaemic rabbit kidney tissue. Int. Urol. Nephrol. 11:325-333.

11. Siegel, N. J., W. B. Glazier, I. H. Chaudry, K. M. Gaudio, B. Lytton, A. E. Baue, and M. Kashgarian. 1980. Enhanced recovery from acute renal failure by the postischemic infusion of adenine nucleotides and magnesium chloride in rats. Kidney Int. 17:338-349.

12. Gaudio, K. M., T. A. Ardito, H. F. Reilly, M. Kashgarian, and N. J. Siegel. 1983. Accelerated cellular recovery after an ischemic renal injury. Am. J. Pathol. 112:338-346.

13. Buhl, M. R. 1976. The postanoxic regeneration of 5'-adenine nucleotides in rabbit tissue during in vitro perfusion. Scand. J. Clin. Lab. Invest. 36:175.

14. Fernando, A. R., J. R. Griffiths, E. P. N. O’Donoghue, J. P. Ward, D. M. G. Armstrong, W. F. Hendry, D. Perrett, and J. E. A. Wickham. 1976. Enhanced preservation of the ischaemic kidney with inosine. Lancet. i:555-557.

15. Rothwell, D., J. Bartley, and M. James. 1981. Preservation of the ischaemic canine kidney with inosine. Urol. Res. 9:75-78.

16. Hanley, M. J., and K. Davidson. 1981. Prior mannitol and furosemide infusion in a model of ischemic acute renal failure. Am. J. Physiol. 241:F556-F564.

17. Burke, T. J., P. E. Arnold, and R. W. Schrier. 1983. Prevention of ischemic acute renal failure with impermeant solutes. Am. J. Physiol. 244:F646-F649.

18. Weinberg, J. M. 1985. Oxygen deprivation-induced injury to isolated rabbit kidney tubules. J. Clin. Invest. 76:1193-1208.

19. Takano, T., S. P. Soltoff, S. Murdaugh, and L. J. Mandel. 1985. Intracellular respiratory dysfunction and cell injury in short-term anoxia of rabbit renal proximal tubules. J. Clin. Invest. 76:2377-2384.

20. Balaban, R. S., S. Soltoff, J. M. Storey, and L. J. Mandel. 1980. Improved renal cortical tubule suspension: spectrophotometric study of $\mathrm{O}_{2}$ delivery. Am. J. Physiol. 238:F50-F59.

21. Soltoff, S. P., and L. J. Mandel. 1984. Active ion transport in the renal proximal tubule. I. Transport and metabolic studies. J. Gen. Physiol. 84:601-622.

22. Mandel, L. J., and E. Murphy. 1984. Regulation of cytosolic free calcium in rabbit proximal renal tubules. J. Biol. Chem. 259:11188-11196.

23. Harris, S. I., R. S. Balaban, L. Barrett, and L. J. Mandel. 1981. Mitochondrial respiratory capacity and $\mathrm{Na}^{+}$- and $\mathrm{K}^{+}$-dependent adenosine triphosphatase-mediated ion transport in the intact renal cell. $J$. Biol. Chem. 256:10319-10328.

24. Layne, E. 1957. Spectrophotometric and turbidimetric methods for measuring proteins. Methods Enzymol. 3:447-454.

25. Bergmeyer, H. U., E. Bernt, and B. Hess. 1963. Lactic dehydrogenase. In Methods of Enzymatic Analysis. H. U. Bergmeyer, editor. Academic Press, Inc., New York. 736-743.

26. Hull-Ryde, E. A., R. G. Cummings, and J. E. Lowe. 1983. Improved method for high energy nucleotide analysis of canine cardiac muscle using reversed-phase high-performance liquid chromatography. J. Chromatogr. 275:411-417.

27. Mandel, L. J., and R. S. Balaban. 1981. Stoichiometry and coupling of active transport to oxidative metabolism in epithelial tissues. Am. J. Physiol. 240:F357-F371.

28. Bagnasco, S., D. Good, R. Balaban, and M. Burg. 1985. Lactate production in isolated segments of the rat nephron. Am. J. Physiol. 248:F522-F526.

29. Aw, T., and D. P. Jones. 1986. Mitochondrial transmembrane distribution of pyruvate and inorganic phosphate $\left(\mathrm{P}_{\mathrm{i}}\right)$ during anoxia. Fed. Proc. 45:1925. (Abstr.) 
30. Pfaller, W., W. G. Guder, G. Gstraunthaler, P. Kotanko, I. Jehart, and S. Purschel. 1984. Compartmentation of ATP within renal proximal tubular cells. Biochim. Biophys. Acta. 805:152-157.

31. Soltoff, S. P., and L. J. Mandel. 1984. Active ion transport in the renal proximal tubule. III. The ATP dependence of the sodium pump. J. Gen. Physiol. 84:643-662.

32. Fox, I. H. 1978. Degradation of purine nucleotides. Handb. Exp. Pharmacol. 51:94-124.

33. Morgan, E. J. 1926. The distribution of xanthine oxidase I. Biochem. J. 20:1282-1291.

34. Richert, D. A., and W. W. Westerfield. 1951. Xanthine oxidases in different species. Proc. Soc. Exp. Biol. Med. 76:252-254.

35. Parker, J. C. 1970. Metabolism of external adenine nucleotides by human red blood cells. Am. J. Physiol. 218:1,568-1,574.

36. Weidemann, M. J., D. A. Hems, and H. A. Krebs. 1969. Effects of added adenine nucleotides on renal carbohydrate metabolism. Biochem. J. 115:1-10.

37. Sumpio, B. E., M. J. Hull, A. E. Bane, and I. H. Chaudry. 1987. Comparison of the effects of ATP- $\mathrm{MgCl}_{2}$ and adenosine- $\mathrm{MgCl}_{2}$ on renal function following ischemia. Am. J. Physiol. 252:R388-R393.
38. Weinberg, J. M., J. A. Davis, A. Lawton, and M. Abarzua. 1988. Modulation of cell nucleotide levels of isolated rabbit kidney tubules. Am. J. Physiol. In press.

39. Sasaki, T., A. Abe, and T. Sakagami. 1983. Ecto-5'-nucleotidase does not catalyze vectorial production of adenosine in the perfused rat liver. J. Biol. Chem. 258:6,947-6,951.

40. Frick, G. P., and J. M. Lowenstein. 1978. Vectorial production of adenosine by 5 '-nucleotidase in the perfused rat heart. J. Biol. Chem. 253:1,240-1,244.

41. Meyskens, F. L., and H. E. Williams. 1971. Adenosine metabolism in human erythrocytes. Biochim. Biophys. Acta. 240:170-179.

42. Le Hir, M., S. Angielski, U. C. Dubach. 1985. Properties of an ecto-5'-nucleotidase of the renal brush border. Renal Physiol. 8:321327.

43. Trimble, M. E., and R. Coulson. 1984. Adenosine transport in perfused rat kidney and renal cortical membrane vesicles. Am. J. Physiol. 84:F794-F803.

44. Le Hir, M., and U. C. Dubach. 1985 . Concentrative transport of purine nucleosides in brush border vesicles of the rat kidney. Eur. J. Clin. Invest. 15:121-127. 